\title{
Interventional Radiologist: Role in Managing Vascular Access Problems in Patients with Chronic Kidney Disease (CKD)
}

\author{
Priyanka Gupta, Pankaj Sharma* and Udit Chauhan \\ Department of Radiodiagnosis, AIIMS Rishikesh, India \\ *Corresponding author: Dr Pankaj Sharma, Associate Professor Radiodiagnosis, AIIMS Rishikesh, India
}

\begin{tabular}{|c|c|}
\hline ARTICLE INFO & ABSTRACT \\
\hline Received: 豐 June 07,2021 & $\begin{array}{l}\text { Citation: Priyanka Gupta, Pankaj Sharma, Udit Chauhan. Interventional Radiologist: Role } \\
\text { in Managing Vascular Access Problems in Patients with Chronic Kidney Disease (CKD). }\end{array}$ \\
\hline Published: 慧 June 16, 2021 & Biomed J Sci \& Tech Res 36(4)-2021. BJSTR. MS.ID.005872. \\
\hline
\end{tabular}

\section{Introduction}

Chronic Kidney disease (CKD) is a common problem in Indian population, and patient depend on vascular access for dialysis, which is done two or three times in a week, with flow rates atleast $600 \mathrm{ml} / \mathrm{min}$. Preferred vascular access for CKD patients is native Arterio-Venous Fistula (AVF), which is surgically created between side of forearm artery and end of vein; with preferred AVF being Radio-Cephalic (RC) fistula, near wrist in non-dependent upper extremity $[1,2]$. If RC fistula can't be surgically created, then other options are Brachio-Cephalic (BC) fistula or Brachio-Basilic (BB) fistula. Less commonly, Arterio-Venous Graft (AVG) may be used for vascular access for CKD patients. These AVG may be straight graft or looped graft. AVG may be between

a) Brachial artery and Cephalic vein

b) Brachial artery and Basilic vein

c) Axillary artery and Axillary vein, or rarely between Common Femoral artery and Great Saphenous vein.

These AVF/AVG take atleast 3-4 weeks to mature. Before AVF or AVG matures, dialysis is done using Tunneled Central Venous Catheter (CVC). Interventional Radiologist (IR) have important role in managing vascular access in patients with CKD. IR secures Tunnelled CVC, preferably through right Internal Jugular vein (IJV), under ultrasound and fluoroscopic guidance, with distal end of catheter, preferably at cavo-atrial junction (Figure 1).
If right IJV is thrombosed, then IR accesses right External Jugular vein (EJV). If right side neck veins are thrombosed, then IR accesses left side neck veins: IJV or EJV (Figure 2). These tunnelled CVC are usually of size 14.5 French, with length 19, 23 or $27 \mathrm{~cm}$, depending on patient's height and from which side, neck vein is to be accessed. The cuff of tunnelled CVC is believed to prevent transmission of infection and also helps in securing CVC. If properly taken care of, then these tunnelled CVC can be used for 6-12 months. IR helps in managing these tunelled CVC, if flow rate is slow, by repositioning these CVC (Figure 3). IR have important role to play in managing AVF with slow flow rates. Most of times, stenosis is seen in these AVF, near anastomotic site. These juxta anastomotic AVF can be managed by IR by Percutaneous Transluminal Angioplasty (PTA), using appropriate size balloon. Some AVF show aneurysmal formation on venous side and these aneurysms can be managed successfully by IR (Figure 4). Sometimes, steal syndrome can be seen in AVF, due to reversal of flow in artery distal to anastomotic site. In case of steal syndrome, IR can block venous side using coils, in case of side to end AVF. This will increase flow rate in distal artery and reduce flow in fistula. In case of side-to-side AVF with steal syndrome, IR can successfully divert blood flow towards distal artery, by blocking collateral draining vein, distal to side-to-side anastomosis (Figure 5). If there is evidence of central vein stenosis, then IR can do PTA of central vein (Figure 6). If there is evidence of elastic recoil, with residual stenosis more than $30 \%$, then IR 
can manage central vein stenosis by using metallic stent (Figure 7). Herein, it is important to stress upon Doppler and Spectral waveform, which helps IR to manage these dialysis fistula and graft [3-5]. In mature fistula and graft, inflow artery shows monophasic flow, with raised Peak systolic velocity (PSV) and End Diastolic velocity (EDV), with spectral broadening (Figure 8). In artery distal to anastomotic site, biphasic or triphasic waveform may be seen. On venous side, spectral waveform shows monophasic flow with turbulent arterial waveform (Figure 9).

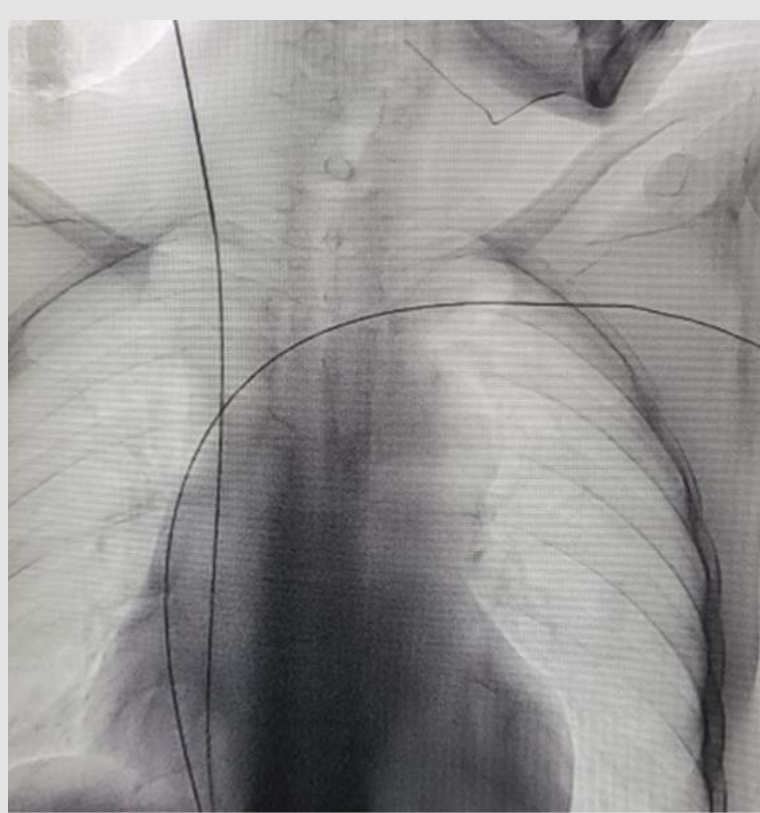

A

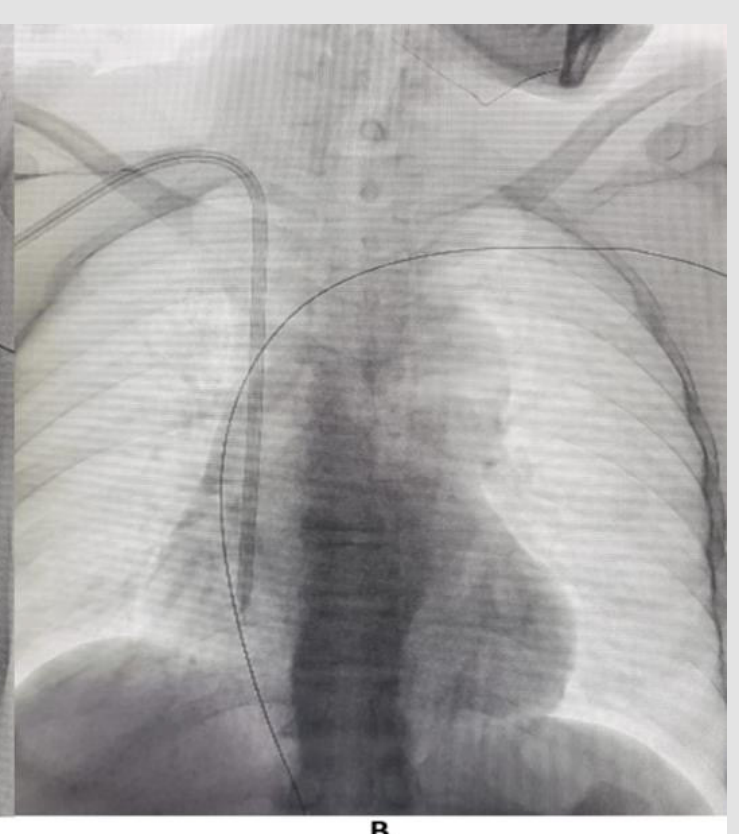

B

\section{Figure 1:}

A. Guidewire in Right internal jugular vein. Also seen is guidewire in central vein, with access from left side.

B. Tunnelled Central Venous Catheter in right internal jugular vein.

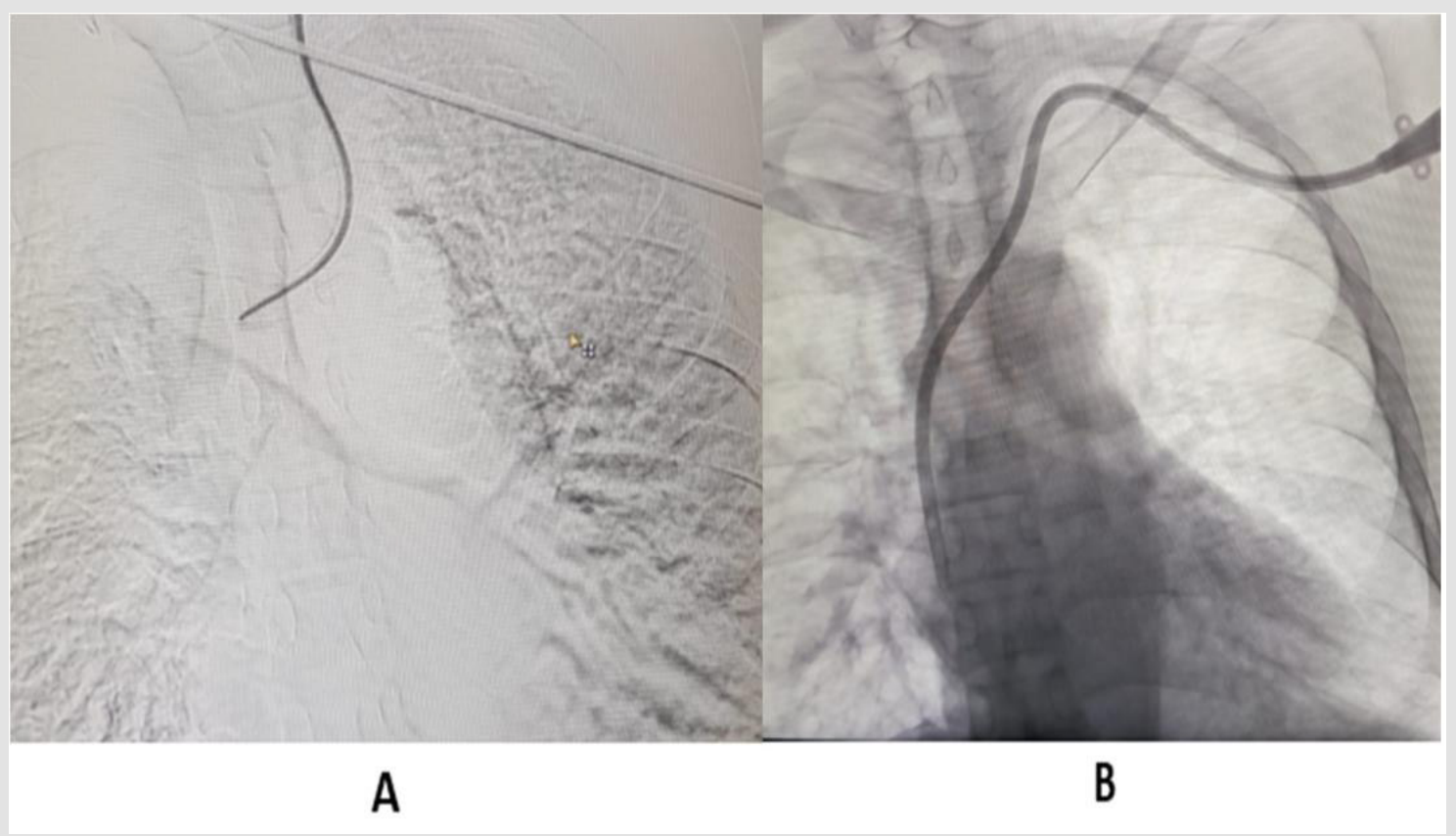

Figure 2:

A. Malpositioned left Tunelled CVC.

B. Tunnelled Central Venous Catheter in left internal jugular vein. 


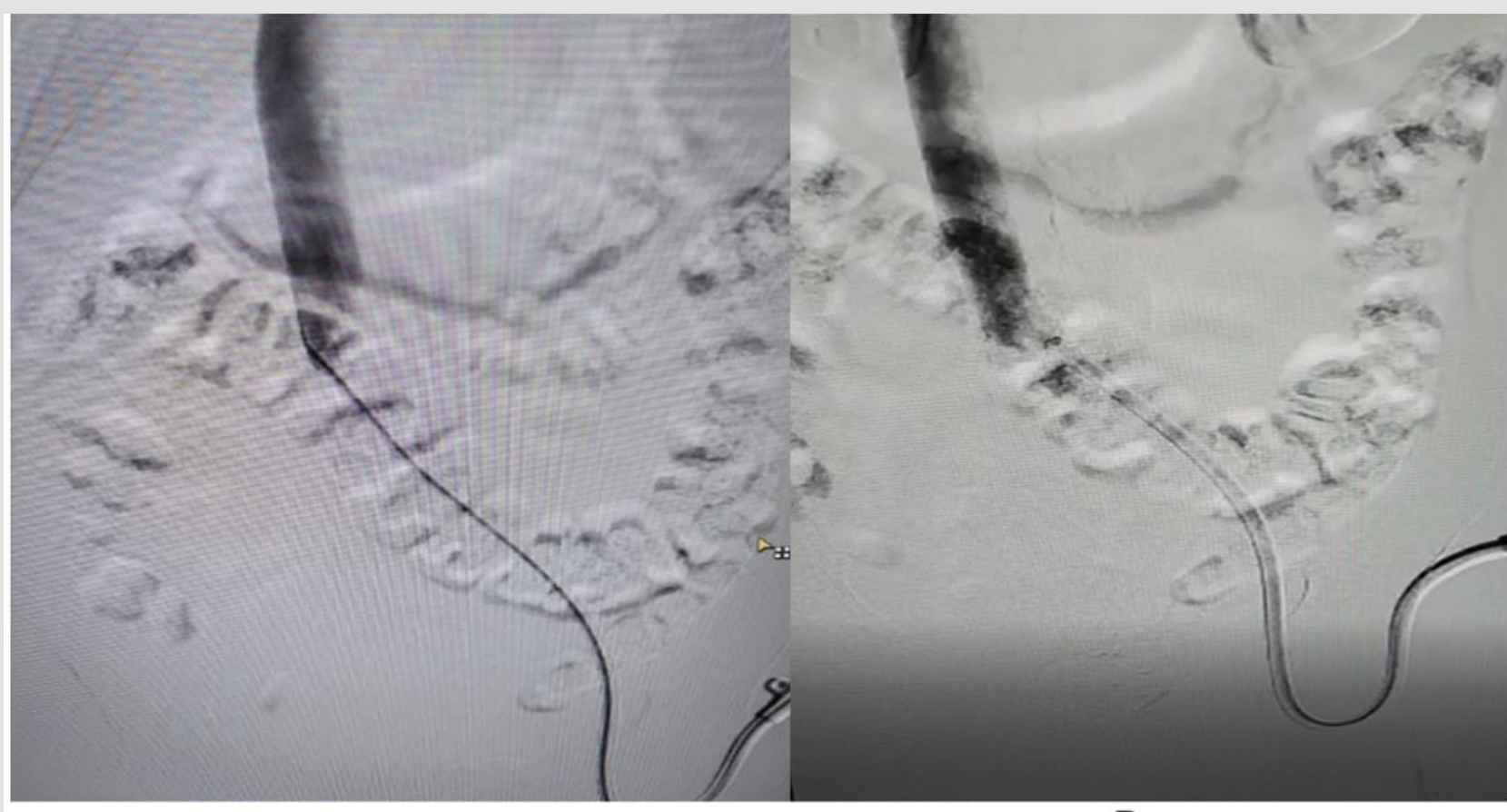

A

B

\section{Figure 3:}

A. Left femoral Tunnelled Central Venous Catheter is not showing free flow of contrast due to fibrin sheath.

B. Tunelled Central Venous Catheter showing free flow of contrast after repositioning.

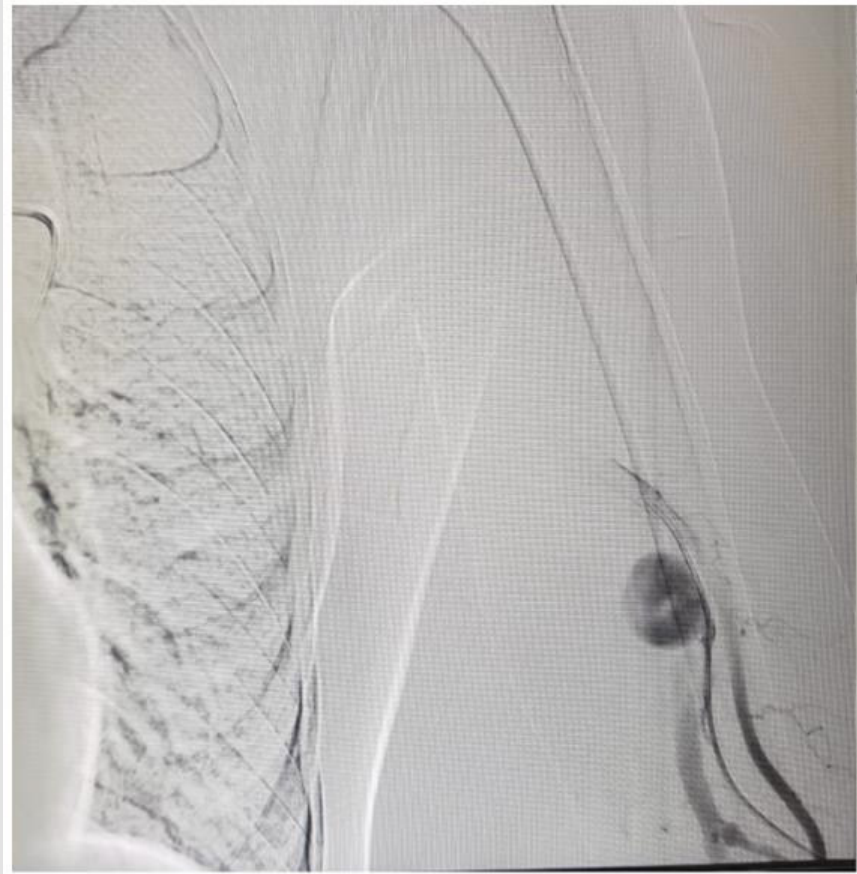

A

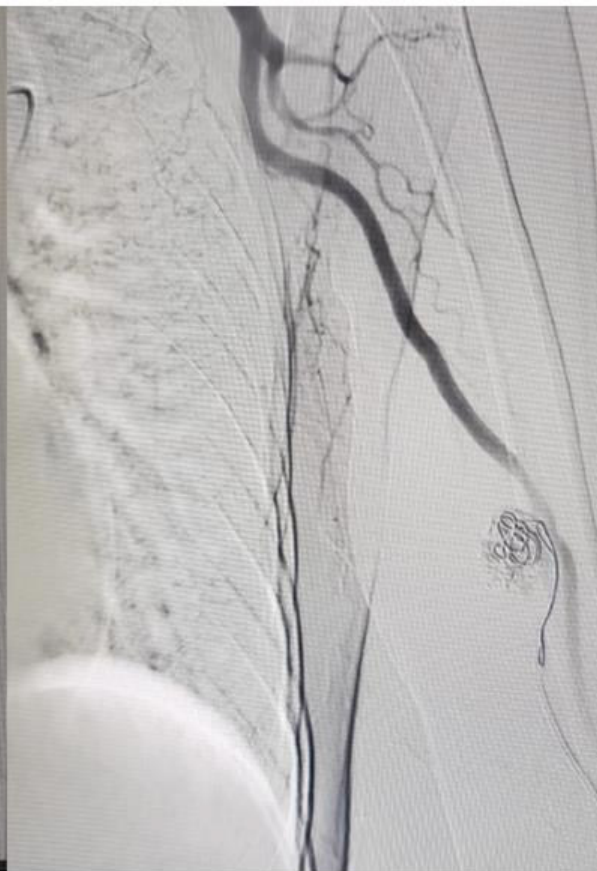

B

\section{Figure 4:}

A. Pseudoaneurysm in draining left brachial vein.

B. Embolization of pseudoaneurysm done using interlock coil. 


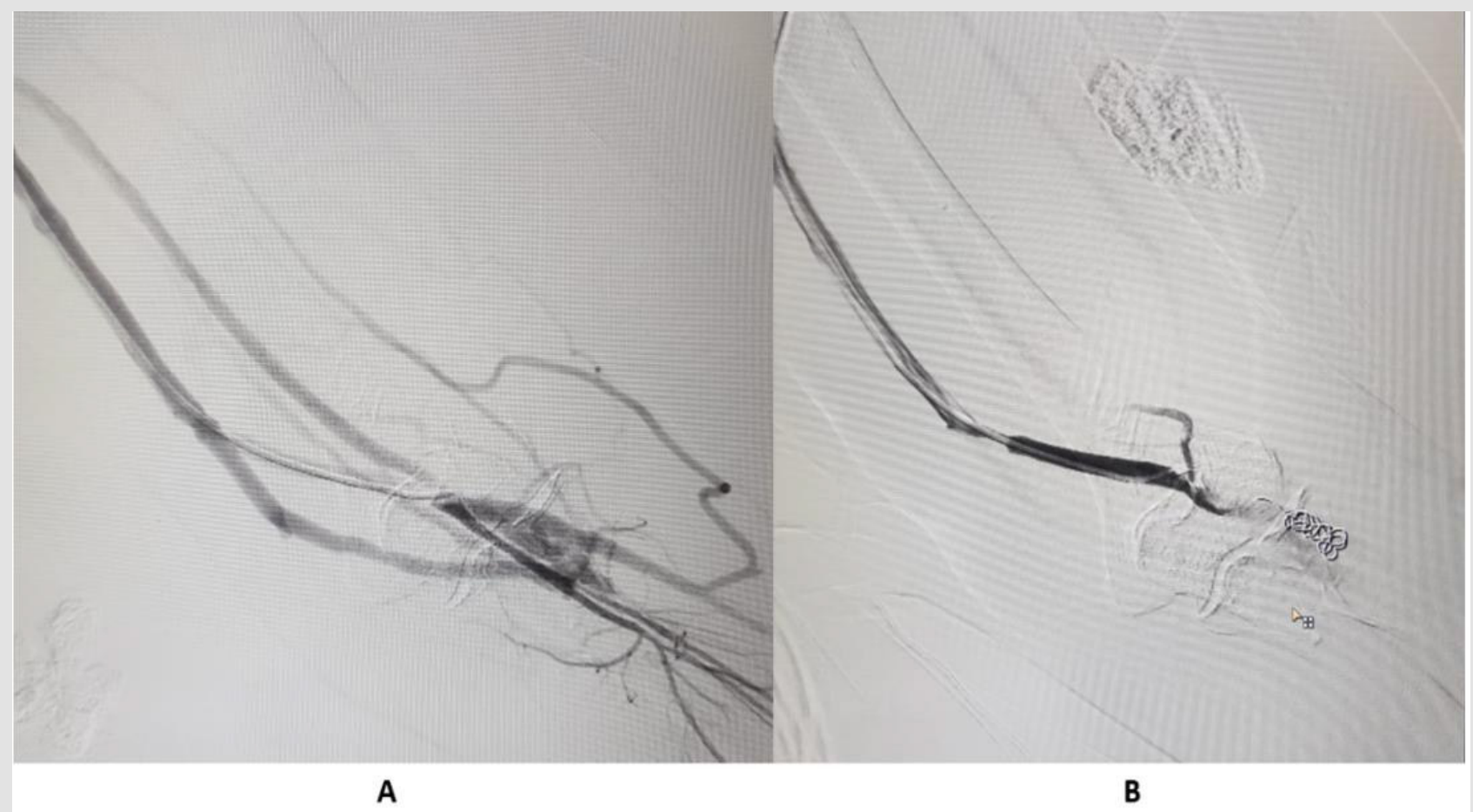

Figure 5:

A. Steal syndrome, with collateral flow.

B. Coil emolization done, with improvement in arterial blood flow in distal forearm and wrist.

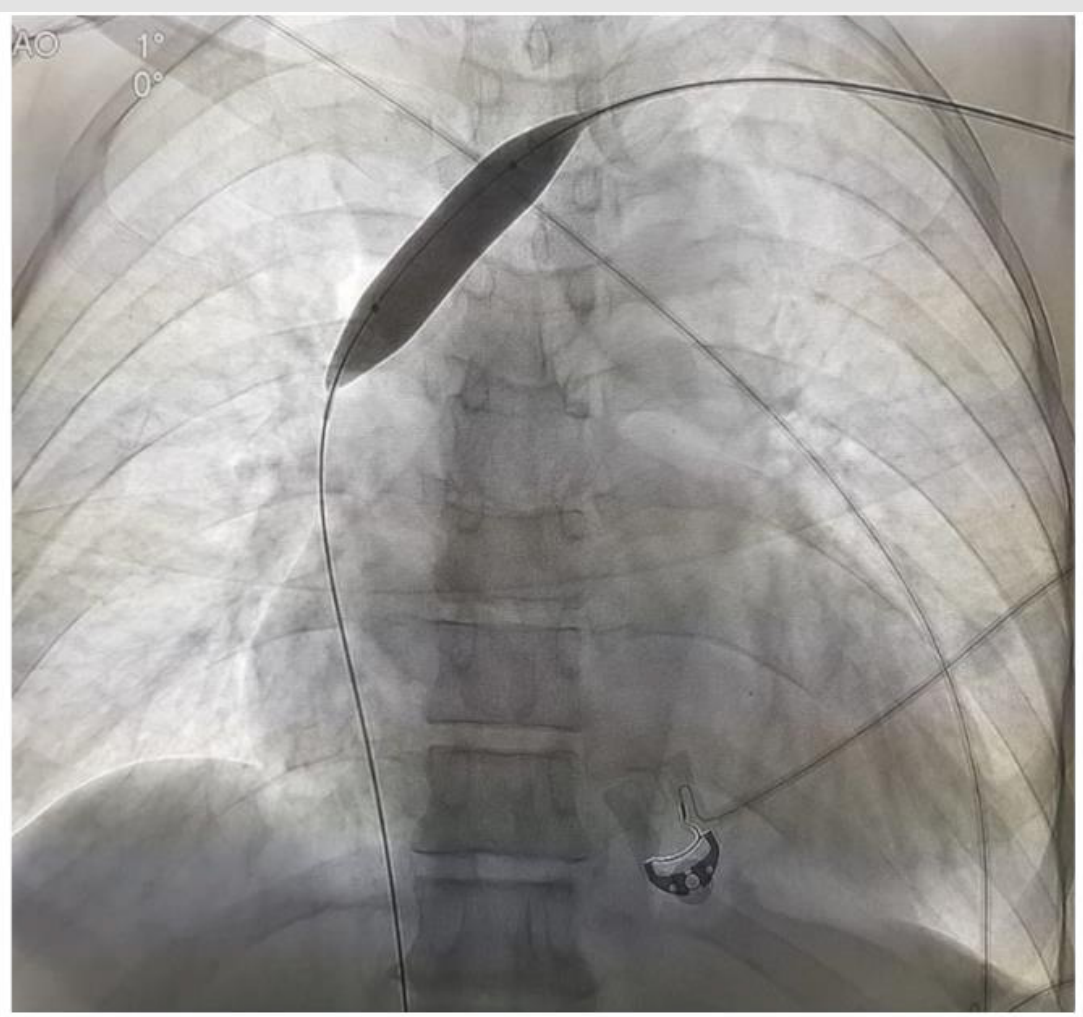

Figure 6: Percutaneous Transluminal Venooplasty left brachiocephalic vein. 


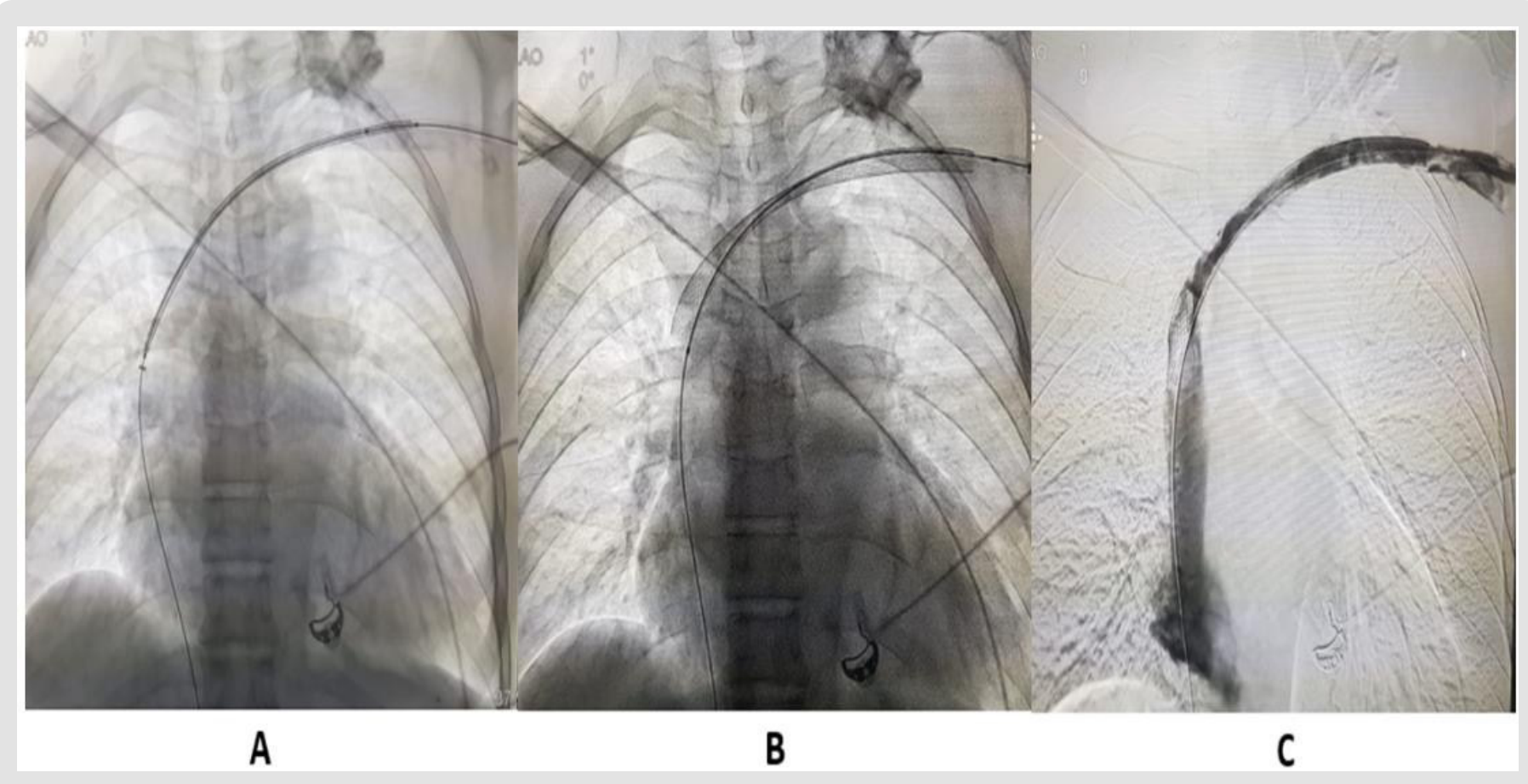

Figure 7:

A. and

B. Metallic stenting left brachiocephalic vein.

C. Post-venoplasty image shows free venous flow of contrast in left brachiocephalic vein.

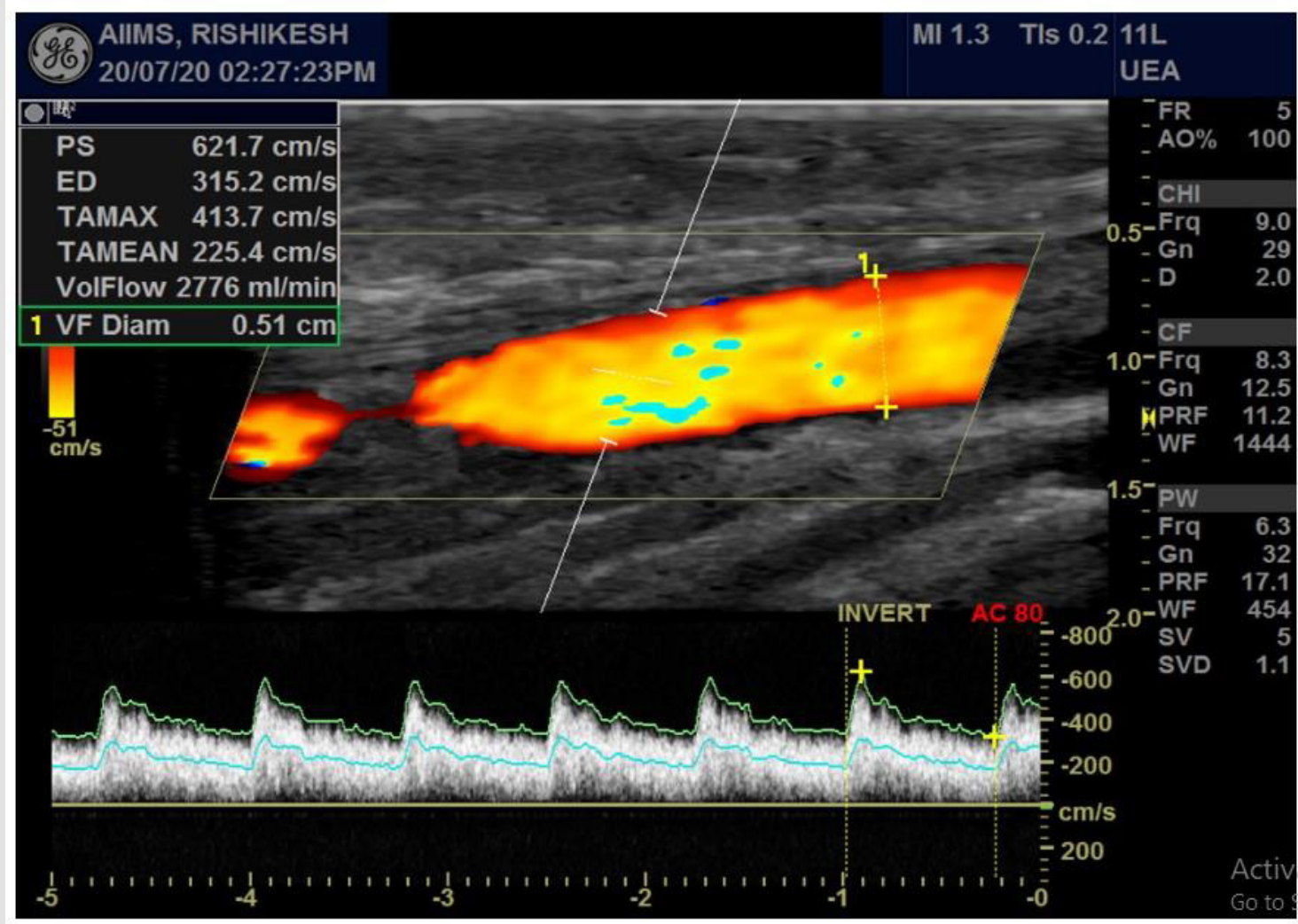

Figure 8: Radio-cephalic fistula with monophasic flow in inflow artery, with raised PSV \& EDV and spectral broadening. 


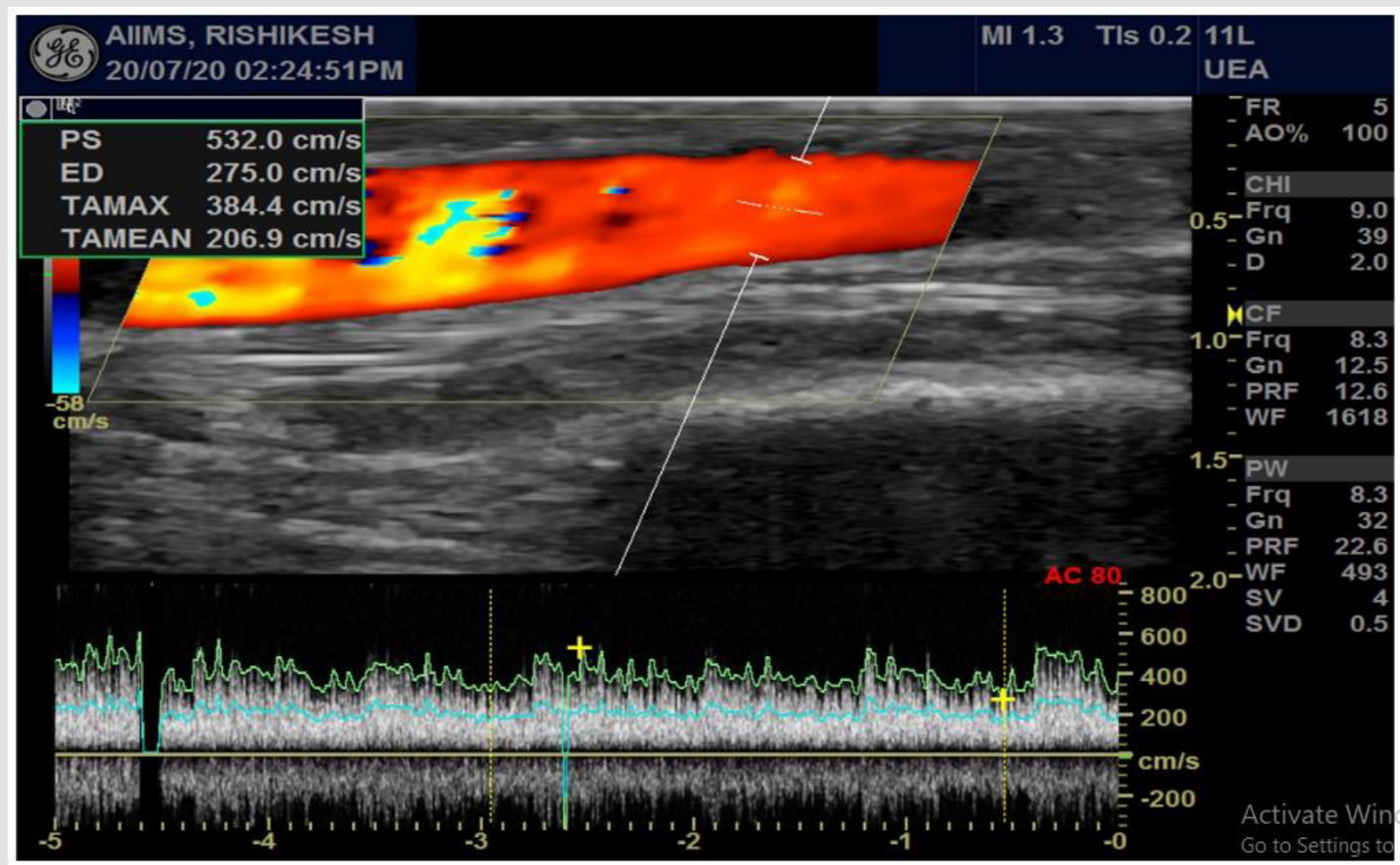

Figure 9: Turbulent monophasic flow with spectral broadening in draining vein.

In immature fistula, inflow artery will show low flow volume. If there is stenosis in AVF, then at site of stenosis, PSV will be high with PSV ratio more than 2. Inflow artery may show triphasic waveform, if fistula occludes. Doppler ultrasound is infact first modality, which an IR relies upon to diagnose, plan and manage, a non-maturing or slowly maturing AVF or AVG. IR can also help in managing thrombosed AVF or AVG. An interesting development is introduction of new device by $\mathrm{BD}$, using which an IR can create AVF, without surgery. But presently this device is very costly, and wide availability is an issue. So, to conclude, IR have become pivotal member of team, managing vascular access problems in patients with CKD.

\section{ISSN: 2574-1241}

DOI: $10.26717 /$ BJSTR.2021.36.005872

Pankaj Sharma. Biomed J Sci \& Tech Res

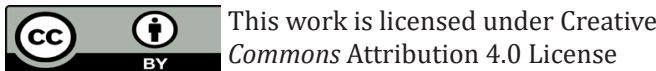

Submission Link: https://biomedres.us/submit-manuscript.php

\section{References}

1. Martin III C, Rex P (2016) Dialysis access anatomy and interventions: a primer. Semin Interven Radiol 33(1): 52-55.

2. Bittl JA (2010) Catheter Interventions for Hemodialysis Fistulas and Grafts. J am Coll Cardiovascular Intervent 3(1): 1-11.

3. Robbin ML, Chamberlain NE, Lockhart ME, Gallichio $\mathrm{MH}$, Carlton J Young, et al. (2002) Hemodialysis arterio-venous fistula maturity: US evaluation. Radiology 225 (1): 59-64.

4. Nalesso F, Garzotto F, Petrucci I, Samoni S, Virzì GM, et al. (2018) Standardized protocol for hemodialysis vascular access assessment: the role of ultrasound and colour doppler. Blood purification 45: 260-269.

5. (2020) AIUM practice parameter for the performance of Vascular Ultrasound Examinations for postoperative assessment of Hemodialysis access. J Ultra Med 9999: 1-10.

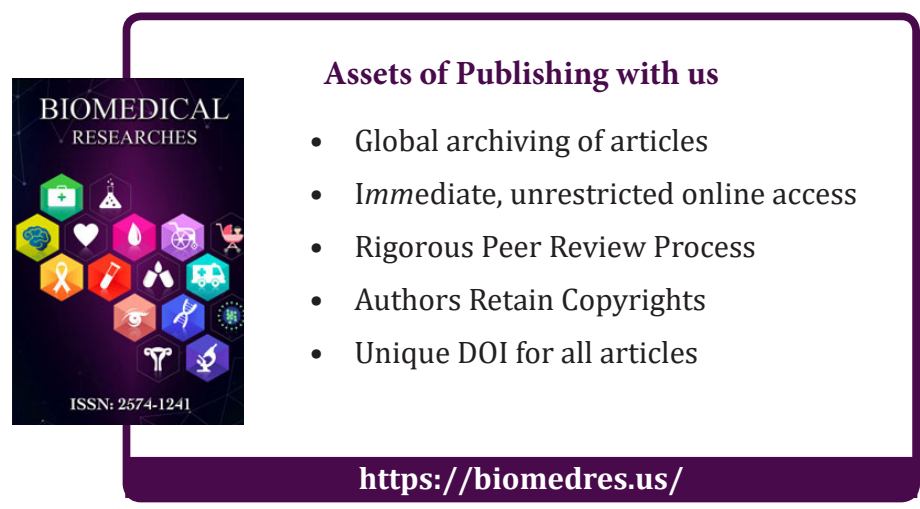

Copyright@ Pankaj Sharma | Biomed J Sci \& Tech Res | BJSTR. MS.ID.005872. 